\title{
Type-2 fuzzy linear systems
}

\author{
Marzieh Najariyan $^{1} \cdot$ Mehran Mazandarani2 $^{2} \cdot$ Robert $\mathrm{John}^{3}$
}

Received: 4 August 2016 / Accepted: 27 December 2016 / Published online: 8 March 2017

(C) Springer International Publishing Switzerland 2017

\begin{abstract}
Fuzzy linear systems (FLSs) are used in practical situations, where some of the systems' parameters or variables are uncertain. To date, investigations conducted on FLSs are restricted to those in which the uncertainty is assumed to be modeled by Type-1 fuzzy sets (T1FSs). However, there are many situations, where considering the uncertainty as T1FSs may not be possible due to different interpretations of experts about the uncertainty. Moreover, solutions of FLSs are T1FSs which do not provide any information about a measure of the dispersion of uncertainty around the T1FSs. Therefore, in this research, a model of uncertain linear equations' system called a type-2 fuzzy linear system is presented to overcome the shortcomings. The uncertainty is represented by a special class of type-2 fuzzy sets-triangular perfect quasi-type-2 fuzzy numbers. In addition, conditions for the existence of a unique type-2 fuzzy solution to the linear system are derived. A definition of a type-2 fuzzy solution is also given. The applicability of the proposed model is illustrated using examples in the pulp and paper industry and electrical engineering.
\end{abstract}

Mehran Mazandarani

me.mazandarani@gmail.com

Marzieh Najariyan

marzieh.najariyan@gmail.com

Robert John

robert.john@nottingham.ac.uk

1 Department of Applied Mathematics Ferdowsi University of Mashhad, Mashhad, Iran

2 Department of Electrical Engineering, Ferdowsi University of Mashhad, Mashhad, Iran

3 LUCID Research Group, School of Computer Science, University of Nottingham, NG8 1BB Nottingham, UK
Keywords Type-2 Fuzzy Numbers · Fuzzy Linear System · Type-2 Fuzzy Sets · Fuzzy Equations

\section{Introduction}

With successful applications in medicine (John and Innocent 2005; Garibaldi et al. 2012; Mazandarani and Kamyad 2011; Najariyan et al. 2011), control theory (Najariyan and Farahi 2015), food science (Zolfaghari et al. 2014), mathematics (Najariyan and Mazandarani 2015), computing with words (Mendel 2016), decision making (Wang et al. 2016; Das et al. 2016), fuzzy logic is well known as an effective tool for dealing with uncertainty in modeling processes. Generalization of concepts and achievements already obtained in the field of mathematics using fuzzy logic have recently captured much attention. Fuzzy linear systems (FLSs) can be regarded as a case of the generalization. By definition, FLSs are systems of linear equations in which coefficients and/or variables are uncertain and this uncertainty is expressed using fuzzy numbers. Simply put, the crisp linear equation system is a special form of an FLS when uncertainty vanishes. Just as the application of linear equations system as a model has been used in various areas (e.g., electrical engineering, chemical, physics), FLS can also enable us to model process and phenomenon more effectively.

FLS was first studied in 1991 (Buckley and QU 1991) and the general framework of FLSs originated in 1998 (Friedman et al. 1998). With the use of the parametric form of Type-1 Fuzzy Numbers (T1FNs) — which are a class of possibility distribution functions-and by replacing the original fuzzy $n \times n$ linear system by a crisp $2 n \times 2 n$ linear system proposed, Friedman et al. (1998) solved FLS whose coefficients matrix was crisp and the right-hand side 
column was an arbitrary T1FN vector. Moreover, it was derived that a unique fuzzy solution does not exist whenever the crisp linear system is not uniquely solved. Then, they continued studying the so-called duality in FLSs (Ming et al. 2000) by considering the linear system as $A X=B X+Y$ in which $A$ and $B$ are real $n \times n$ matrices, $X$ and $Y$ are unknown and known vectors of T1FNs, respectively. It was demonstrated that the dual FLS has a unique fuzzy solution on conditions that the inverse matrix of $A-B$ exists and has only non-negative entries.

Since then, extensive research was conducted on the issue with several methods for solving FLSs presented, such as the steepest descent method (Abbasbandy and Jafarian 2006), LU decomposition method (Abbasbandy et al. 2006), perturbation analysis (Tian et al. 2010), and the linear programming problem approach (Ghanbari 2015). In addition, some studies were dedicated to investigate different forms of FLSs. In (Wang et al. 2001), solving FLS $X=A X+U$, where $A$ is a crisp square matrix, and $U$ a vector of fuzzy numbers was considered. A method for solving fuzzy linear systems of the form $A_{1} X+b_{1}=A_{2} X+b_{2}$ -in which $A_{1}$ and $A_{2}$ are fuzzy square matrices, and $b_{1}$ and $b_{2}$ are fuzzy numbers vectors-was proposed in Muzzioli and Reynaerts (2006). Furthermore, obtaining a solution to fuzzy complex system of linear equations was investigated in Behera and Chakraverty (2014).

To date, all studies dealing with FLSs are restricted to those where uncertainty is considered as a T1FN. In other words, it has been assumed that the uncertainty can be determined using a precise membership function. However, it may not be possible for a decision maker to consider such an exact form based on different experts' interpretations of the uncertainty in real applications. To illustrate, suppose a number of electrical engineers-as the experts—are asked about the amount of output voltage of a specific amplifier system. All the subjects mention "approximately 10 volts". Nevertheless, if each individual subject is asked to show the "approximately 10 volts membership function" as T1FN, different T1FNs are likely to be presented, even if the T1FNs are all of the same kind (e.g., triangular). This issue recalls the statement that words can mean different things to different people (Mendel 2007). The motivation for this paper comes from this observation that determining an exact form of one single possibility distribution function of an uncertain value may not be always possible based on different experts' interpretations about the value.

In 1975 (Zadeh 1975), Zadeh presented Type-2 Fuzzy Sets (T2FSs) which are a more general concept than Type-1 Fuzzy Sets (T1FSs). By definition, a T2FS is a set in which membership grades are a T1FS each. This paper aims at investigating FLSs in which uncertainty is considered as a special class of T2FSs called triangular perfect quasi-type-2 fuzzy numbers (Mazandarani and Najariyan 2014b). This system has a solution which includes not only different interpretations of experts, but also provides information about uncertainty dispersion. This paper also presents conditions for the existence of a unique fuzzy solution to the $n \times n$ linear system. Furthermore, the applicability of the proposed model is illustrated using examples in the pulp and paper industry, and electrical engineering.

\section{Preliminaries}

This section presents some necessary definitions and theorems which will be used in this paper.

Throughout this paper, the set of all real numbers is denoted by $\mathbb{R}$, the set of all T1FNs on $\mathbb{R}$ by $E_{1}$, and the set of all perfect T2FNs on $\mathbb{R}$ by $E_{2}$. The left and right endpoints of $\alpha$-cut of a fuzzy set $A, A^{\alpha}$, are denoted by $A^{\alpha}$ and $\bar{A}^{\alpha}$, respectively. The transpose of a matrix $Y=\left[y_{i j}\right]_{n \times n}$ is denoted by $Y^{T}$.

Definition 1 (Zimmermann 2001) The T1FS $u: \mathbb{R} \rightarrow[0,1]$ is called a T1FN if it is normal, fuzzy convex, upper semi-continuous and compactly supported fuzzy subsets of the real numbers.

The T1FN $u \in E_{1}$ can be represented in a parametric form by the ordered pair of functions $\left(\underline{u}^{\alpha}, \bar{u}^{\alpha}\right), 0 \leq \alpha \leq 1$ satisfying the following properties:

1. $\underline{u}^{\alpha}$ is a bounded non-decreasing left continuous function;

2. $\bar{u}^{\alpha}$ is a bounded non-increasing left continuous function;

3. $\underline{u}^{\alpha} \leq \bar{u}^{\alpha}, \alpha \in[0,1]$.

Definition 2 (Mendel 2008; Coupland and John 2007) A T2FS, $\tilde{A}$, can be characterized by the so-called point-valued representation as follows:

$\tilde{A}=\left\{\left((x, u), \mu_{\tilde{A}}(x, u)\right) \mid \forall x \in X, \forall u \in J_{x} \subseteq[0,1]\right\}$,

where $\mu_{\tilde{A}}(x, u)$ and $u$ are called type- 2 membership function and primary grade - or secondary variable - of $\tilde{A}$, respectively, $x$ is the primary variable, $X$ is the domain of the fuzzy set, and $J_{x}$ is the domain of the secondary membership function at $x$ (see Fig. 1).

Definition 3 (Mendel and John 2002). Let $\tilde{A}$ be a T2FS. A vertical slice of $\tilde{A}$ at $x_{0} \in X, \mu_{\tilde{A}}\left(x_{0}\right)$, is a T1FS and the membership function of secondary domain at the fixed point $x_{0}$. It is identified as 
Fig. 1 Triangular type-2 fuzzy set with Lower Membership Function (LMF), Upper Membership Function (UMF), and Principle Set (PS). Top insert depicts the secondary membership function, i.e., vertical slice at $x_{0}$

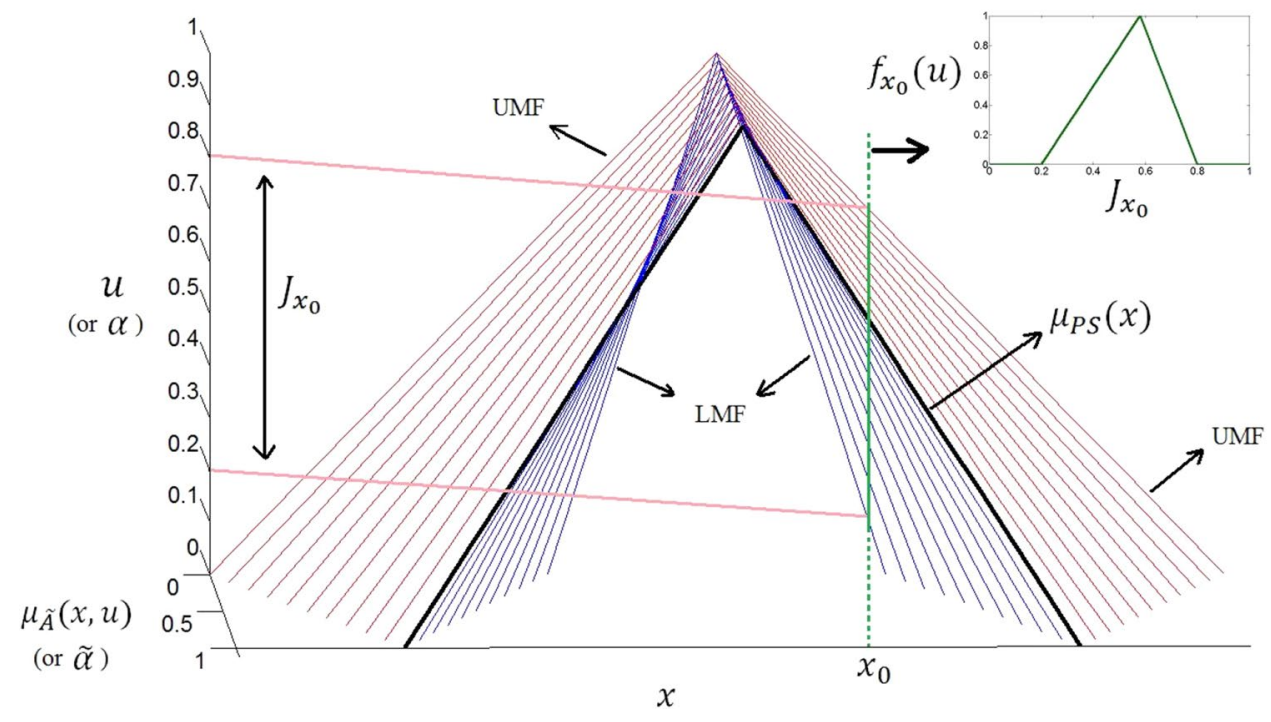

$\mu_{\tilde{A}}\left(x_{0}\right)=\int_{u \in J_{x_{0}}} f_{x_{0}}(u) / u$

where is called secondary grade.

It should be noted that the integral sign stands for the union over all admissible $x$ and/or $u$ is not standard integration. In addition, the sign "/" stands for association (or a marker) does not imply division.

Definition 4 (Mazandarani and Najariyan 2014a). An $\tilde{\alpha}$ cut set of the vertical slice of $\tilde{A}$ at point $x_{0} \in X$ is defined as

$S_{\tilde{A}}\left(x_{0} \mid \tilde{\alpha}\right)= \begin{cases}\left\{u \in J_{x_{0}} \mid f_{x_{0}}(u) \geq \tilde{\alpha}\right\}, & 0<\tilde{\alpha} \leq 1, \\ c l\left\{u \in J_{x_{0}} \mid f_{x_{0}}(u)>\tilde{\alpha}\right\}, & \tilde{\alpha}=0,\end{cases}$

where $c l($.$) denotes the closure of the set, and it is compact.$

Definition 5 (Liu 2008) Let $\tilde{A}$ be a T2FS. The union of all secondary domains of the T2FS, whose secondary grades are greater or equal to $\tilde{\alpha} \in[0,1]$, is called $\tilde{\alpha}$-plane of $\tilde{A}$ and denoted by $\tilde{A}_{\tilde{\alpha}}$ as

$\tilde{A}_{\tilde{\alpha}}=\int_{x \in X} \int_{u \in J_{x}}\left\{(x, u) \mid S_{\tilde{A}}(x \mid \tilde{\alpha})\right\}$

$\tilde{A}_{\tilde{\alpha}}=\int_{x \in X} \int_{u \in J_{x}}\left\{(x, u) \mid f_{x}(u) \geq \tilde{\alpha}\right\}$

Theorem 1 (Liu 2008) $\tilde{\alpha}$-plane representation theorem: A T2FS, $\tilde{A}$, can be represented as the union of its $\tilde{\alpha}$-planes, i.e., $\tilde{A}=\bigcup_{\tilde{\alpha} \in[0,1]} \tilde{\alpha} \tilde{A}_{\tilde{\alpha}}$.

Definition 6 (Mazandarani and Najariyan 2014a; Hung and Yang 2004). Let $\tilde{A}$ and $\tilde{B}$ be two type-2 fuzzy sets, and
$d_{H}$ denote the well-known Hausdorff distance. A metric on the space of type-2 fuzzy sets is defined as follows:

$d_{2}(\tilde{A}, \tilde{B})=\int_{a}^{b} H_{f}\left(\mu_{\tilde{A}}(x), \mu_{\tilde{B}}(x)\right) d x$

where

$H_{f}\left(\mu_{\tilde{A}}(x), \mu_{\tilde{B}}(x)\right)=2 \int_{0}^{1} \tilde{\alpha} d_{H}\left(S_{\tilde{A}}(x \mid \tilde{\alpha}), S_{\tilde{B}}(x \mid \tilde{\alpha})\right) d \tilde{\alpha}$

Definition 7 (Mazandarani and Najariyan 2014a; Mendel et al. 2009) Suppose $\tilde{A}$ is a T2FS. The $\tilde{\alpha}$-plane of the set at $\tilde{\alpha}=0, \tilde{A}_{0}$, is called Footprint of Uncertainty of $\tilde{A}, \operatorname{FOU}(\tilde{A})$. In other words, $\mathrm{FOU}(\tilde{A})$ is a bounded region of the set when it is mapped on two-dimensional plane in the $x$ and $u$ axes.

Note 1 The $\tilde{\alpha}$-plane of a T2FS, $\tilde{A}_{\tilde{\alpha}}$, is an interval valued fuzzy set, i.e., it is a set all of whose elements have interval membership grades (Hamrawi 2011). That is, the T2FS at level $\tilde{\alpha}, \tilde{A}_{\tilde{\alpha}}$, can be characterized in a parametric form by the pair $\left(\underline{A}_{\tilde{\alpha}}, \bar{A}_{\tilde{\alpha}}\right)$, where $\underline{A}_{\tilde{\alpha}}$ is called Lower Membership Function [LMF], and $\bar{A}_{\tilde{\alpha}}$ is called Upper Membership Function [UMF], and they are both T1FSs (Mazandarani and Najariyan 2014a).

Definition 8 (Hamrawi 2011) Let $\tilde{A}$ be a T2FS. The $\tilde{\alpha}$ plane of the set at $\tilde{\alpha}=1, \tilde{A}_{1}$, is called Principle Set [PS] of $\tilde{A}$, and its membership function is defined as

$\mu_{P S}(x)=\int_{x \in X} u / x$ s.t. $f_{x}(u)=1$ 
Definition 9 (Hamrawi 2011; Hamrawi et al. 2010) Suppose $\tilde{A}$ is a T2FS and its $\tilde{\alpha}$-plane is $\tilde{A}_{\tilde{\alpha}}=\left(\underline{A}_{\tilde{\alpha}}, \bar{A}_{\tilde{\alpha}}\right)$. The $\alpha$-cut of the $\tilde{\alpha}$-plane, $\tilde{A}_{\tilde{\alpha}}^{\alpha}$, is the $\alpha$-cut of its LMF and UMF, i.e., $\tilde{A}_{\tilde{\alpha}}^{\alpha}=\left(\underline{A}_{\tilde{\alpha}}^{\alpha}, \bar{A}_{\tilde{\alpha}}^{\alpha}\right)$.

Theorem 2 (Hamrawi 2011; Hamrawi et al. 2010) A $T 2 F S, \tilde{A}$, can be represented by the union of all itso-cuts as follows:

$$
\tilde{A}=\bigcup_{\tilde{\alpha} \in[0,1]} \tilde{\alpha} \bigcup_{\alpha \in[0,1]} \alpha \tilde{A}_{\tilde{\alpha}}^{\alpha}
$$

\section{Triangular perfect quasi-type-2 fuzzy numbers}

Definition 10 (Hamrawi 2011; Hamrawi et al. 2016; Mazandarani and Najariyan 2014b) A T2FS, $\tilde{A}$, is called a perfect $\mathrm{T} 2 \mathrm{FN}$ if the following conditions are satisfied:

1. UMF and LMF of FOU $(\tilde{A})$ are T1FNs themselves;

2. UMF and LMF of PS of $\tilde{A}$ are T1FNs themselves.

Definition 11 (Hamrawi 2011; Hamrawi et al. 2016) A perfect QT2FN is a perfect T2FN, whose all vertical slices are T1FNs and piecewise functions are of the same kind (e.g., linear).

Note 2 (Hamrawi 2011) A perfect QT2FN can be completely determined using its FOU and PS.
A class of triangular perfect QT2FNs was introduced for the first time by Mazandarani and Najariyan in Mazandarani and Najariyan (2014b) as the septuple:

$\tilde{w}=\left(\bar{L}_{w_{0}}, L_{w_{1}}, \underline{L}_{w_{0}}, m, \underline{R}_{w_{0}}, R_{w_{1}}, \bar{R}_{w_{0}}\right)$

where $\bar{L}_{w_{0}} \leq L_{w_{1}} \leq \underline{L}_{w_{0}} \leq m \leq \underline{R}_{w_{0}} \leq R_{w_{1}} \leq \bar{R}_{w_{0}}$. Figure 2 shows the triangular perfect QT2FNs. The triangular perfect QT2FN, $\tilde{w}$, in the levels $\alpha$ and $\tilde{\alpha}, \tilde{w}_{\tilde{\alpha}}^{\alpha}=\left(\underline{w}_{\tilde{\alpha}}^{\alpha}, \bar{w}_{\tilde{\alpha}}^{\alpha}\right)$, is determined as

$\left\{\begin{array}{l}\bar{w}_{\tilde{\alpha}}^{\alpha}=\left[\bar{L}_{w_{\tilde{\alpha}}}^{\alpha}, \bar{R}_{w_{\tilde{\alpha}}}^{\alpha}\right] \\ \bar{L}_{w_{\tilde{\alpha}}}^{\alpha}=L_{w_{1}}^{\alpha}-(1-\tilde{\alpha})\left(L_{w_{1}}^{\alpha}-\bar{L}_{w_{0}}^{\alpha}\right) \\ \bar{R}_{w_{\tilde{\alpha}}}^{\alpha}=R_{w_{1}}^{\alpha}-(1-\tilde{\alpha})\left(R_{w_{1}}^{\alpha}-\bar{R}_{w_{0}}^{\alpha}\right)\end{array}\right.$

and

$\left\{\begin{array}{l}\underline{w}_{\tilde{\alpha}}^{\alpha}=\left[\underline{L}_{w_{\tilde{\alpha}}}^{\alpha}, \underline{R}_{w_{\tilde{\alpha}}}^{\alpha}\right] \\ \underline{L}_{w_{\tilde{\alpha}}}^{\alpha}=L_{w_{1}}^{\alpha}-(1-\tilde{\alpha})\left(L_{w_{1}}^{\alpha}-\underline{L}_{w_{0}}^{\alpha}\right) \\ \underline{R}_{w_{\tilde{\alpha}}}^{\alpha}=R_{w_{1}}^{\alpha}-(1-\tilde{\alpha})\left(R_{w_{1}}^{\alpha}-\underline{R}_{w_{0}}^{\alpha}\right)\end{array}\right.$

where $\bar{L}_{w_{0}}^{\alpha} \leq L_{w_{1}}^{\alpha} \leq \underline{L}_{w_{0}}^{\alpha} \leq \underline{R}_{w_{0}}^{\alpha} \leq R_{w_{1}}^{\alpha} \leq \bar{R}_{w_{0}}^{\alpha}$, and they are characterized as

$\left\{\begin{array}{l}\underline{L}_{w_{0}}^{\alpha}=m-(1-\alpha)\left(m-\underline{L}_{w_{0}}\right) \\ \underline{R}_{w_{0}}^{\alpha}=m-(1-\alpha)\left(m-\underline{R}_{w_{0}}\right) \\ \bar{L}_{w_{0}}^{\alpha}=m-(1-\alpha)\left(m-\bar{L}_{w_{0}}\right) \\ \bar{R}_{w_{0}}^{\alpha}=m-(1-\alpha)\left(m-\bar{R}_{w_{0}}\right) \\ L_{w_{1}}^{\alpha}=m-(1-\alpha)\left(m-L_{w_{1}}\right) \\ R_{w_{1}}^{\alpha}=m-(1-\alpha)\left(m-R_{w_{1}}\right) .\end{array}\right.$

In the following, some of the arithmetic operations on the triangular perfect QT2FNs are given. Let
Fig. 2 Triangular perfect QT2FN, $\tilde{w}$, with $\underline{L}_{w_{0}}, \underline{R}_{w_{0}}$ as the left and right endpoints of the support of LMF, $\bar{L}_{w_{0}}, \bar{R}_{w_{0}}$ as the left and right endpoints of the support of UMF, $L_{w_{1}}, R_{w_{1}}$ as the left and right endpoints of the support of PS and $m$ as the core of the set

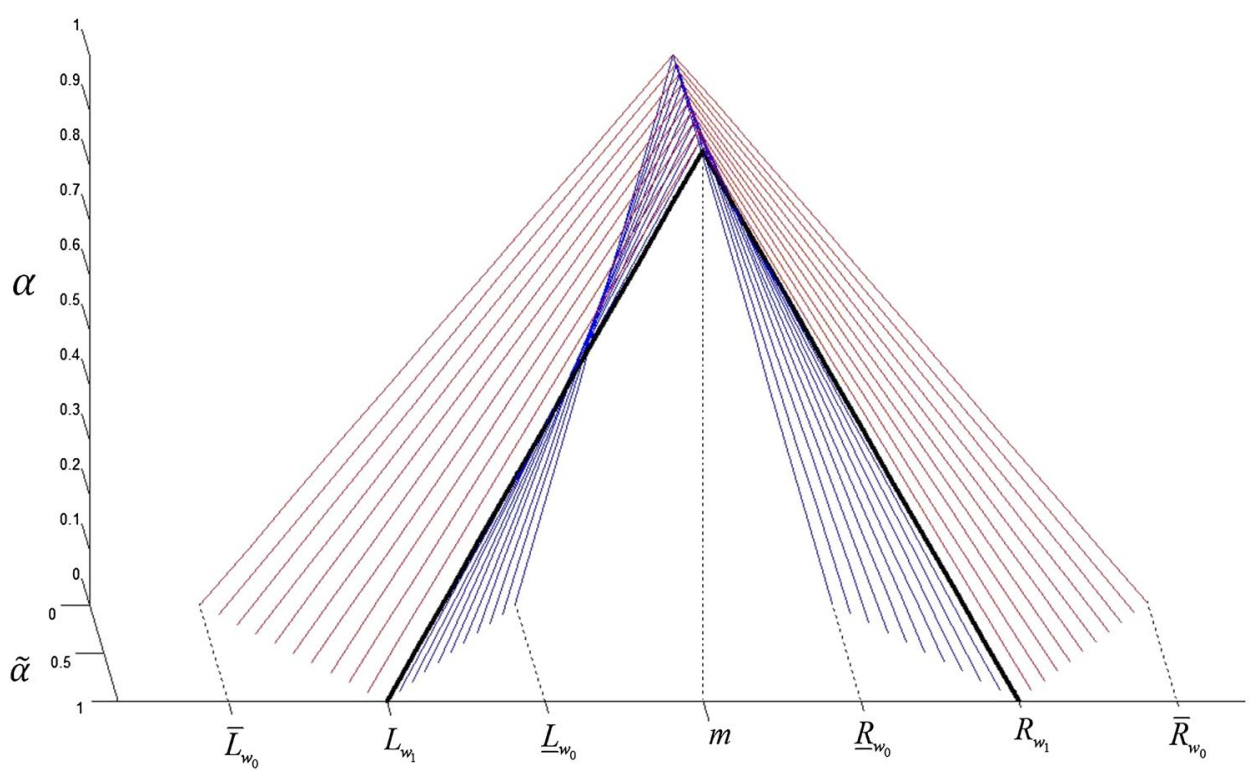


$\tilde{w}=\left(\bar{L}_{w_{0}}, L_{w_{1}}, \underline{L}_{w_{0}}, m, \underline{R}_{w_{0}}, R_{w_{1}}, \bar{R}_{w_{0}}\right)$ and $\tilde{z}=\left(\bar{L}_{z_{0}}, L_{z_{1}}, \underline{L}_{z_{0}}, n\right.$, $\underline{R}_{z_{0}}, R_{z_{1}}, \bar{R}_{z_{0}}$ ) be two triangular perfect QT2FNs. The addition, $\tilde{w}+\tilde{z}$, and scalar multiplication by $k \in \mathbb{R}, k \tilde{w}$, are defined as follows:

$$
\begin{gathered}
\tilde{w}+\tilde{z}=\left(\bar{L}_{w_{0}}+\bar{L}_{z_{0}}, L_{w_{1}}+L_{z_{1}}, \underline{L}_{w_{0}}+\underline{L}_{z_{0}}, m+n,\right. \\
\left.\underline{R}_{w_{0}}+\underline{R}_{z_{0}}, R_{w_{1}}+R_{z_{1}}, \bar{R}_{w_{0}}+\bar{R}_{w_{0}}\right)
\end{gathered}
$$

$k \tilde{w}=\left(k \bar{L}_{w_{0}}, k L_{w_{1}}, k \underline{L}_{w_{0}}, k m, k \underline{R}_{w_{0}}, k R_{w_{1}}, k \bar{R}_{w_{0}}\right)$

for $k \geq 0$,

$k \tilde{w}=\left(k \bar{R}_{w_{0}}, k R_{w_{1}}, k \underline{R}_{w_{0}}, k m, k \underline{L}_{w_{0}}, k L_{w_{1}}, k \bar{L}_{w_{0}}\right)$

for $k<0$.

Moreover, the scalar multiplication by $k \in \mathbb{R}$, addition, subtraction, and multiplication, in the levels $\alpha$ and $\tilde{\alpha}$ is characterized as

$[k \tilde{w}]_{\tilde{\alpha}}^{\alpha}=\left\{\begin{array}{l}\left(\left[k \underline{L}_{w_{\tilde{\alpha}}}^{\alpha}, k \underline{R}_{w_{\tilde{\alpha}}}^{\alpha}\right],\left[k \bar{L}_{w_{\tilde{\alpha}}}^{\alpha}, k \bar{R}_{w_{\tilde{\alpha}}}^{\alpha}\right]\right), k \geq 0, \\ \left(\left[k \underline{R}_{w_{\tilde{\alpha}}}^{\alpha}, k{\underline{L_{w}}}_{w_{\tilde{\alpha}}}^{\alpha}\right],\left[k \bar{R}_{w_{\tilde{\alpha}}}^{\alpha}, k \bar{L}_{w_{\tilde{\alpha}}^{\alpha}}^{\alpha}\right], k<0\right.\end{array}\right.$

and

$[\tilde{w} \circ \tilde{z}]_{\tilde{\alpha}}^{\alpha}=\left(\left[\underline{w}_{\tilde{\alpha}}^{\alpha} \circ z_{\tilde{\alpha}}^{\alpha}\right],\left[\bar{w}_{\tilde{\alpha}}^{\alpha} \circ \bar{z}_{\tilde{\alpha}}^{\alpha}\right]\right)$

where $\tilde{w} \circ \tilde{z}$ means $\tilde{w}+\tilde{z}$ or $\tilde{w}-\tilde{z}$ or $\tilde{w} \times \tilde{z}$ and

$$
\begin{aligned}
{\left[\underline{w}_{\tilde{\alpha}}^{\alpha} \circ \underline{z}_{\tilde{\alpha}}^{\alpha}\right]=} & {\left[\operatorname { m i n } \left\{\underline{L}_{w_{\tilde{\alpha}}}^{\alpha} \circ \underline{L}_{z_{\tilde{\alpha}}}^{\alpha}, \underline{L}_{w_{\tilde{\alpha}}}^{\alpha} \circ \underline{R}_{z_{\tilde{\alpha}}}^{\alpha}, \underline{R}_{w_{\tilde{\alpha}}}^{\alpha} \circ \underline{L}_{z_{\tilde{\alpha}}}^{\alpha},\right.\right.} \\
& \left.\underline{R}_{w_{\tilde{\alpha}}}^{\alpha} \circ \underline{R}_{z_{\tilde{\alpha}}}^{\alpha}\right\}, \\
& \max \left\{\underline{L}_{w_{\tilde{\alpha}}} \circ \underline{L}_{z_{\tilde{\alpha}}}, \underline{L}_{w_{\tilde{\alpha}}} \circ \underline{R}_{z_{\tilde{\alpha}}}, \underline{R}_{w_{\tilde{\alpha}}} \circ \underline{L}_{z_{\tilde{\alpha}}},\right. \\
& \left.\left.\underline{R}_{w_{\tilde{\alpha}}} \circ \underline{R}_{z_{\tilde{\alpha}}}\right\}\right] \\
{\left[\bar{w}_{\tilde{\alpha}}^{\alpha} \circ \bar{z}_{\tilde{\alpha}}^{\alpha}\right]=} & {\left[\operatorname { m i n } \left\{\bar{L}_{w_{\tilde{\alpha}}^{\alpha}}^{\alpha} \circ \bar{L}_{z_{\tilde{\alpha}}}^{\alpha}, \bar{L}_{w_{\tilde{\alpha}}}^{\alpha} \circ \bar{R}_{z_{\tilde{\alpha}}}^{\alpha}, \bar{R}_{w_{\tilde{\alpha}}}^{\alpha} \circ \bar{L}_{z_{\tilde{\alpha}}}^{\alpha},\right.\right.} \\
& \left.\bar{R}_{w_{\tilde{\alpha}}^{\alpha}}^{\alpha} \circ \bar{R}_{z_{\tilde{\alpha}}}^{\alpha}\right\}, \\
& \max \left\{\bar{L}_{w_{\tilde{\alpha}}} \circ \bar{L}_{z_{\tilde{\alpha}}}, \bar{L}_{w_{\tilde{\alpha}}} \circ \bar{R}_{z_{\tilde{\alpha}}}, \bar{R}_{w_{\tilde{\alpha}}} \circ \bar{L}_{z_{\tilde{\alpha}}},\right. \\
& \left.\left.\bar{R}_{w_{\tilde{\alpha}}} \circ \bar{R}_{z_{\tilde{\alpha}}}\right\}\right] .
\end{aligned}
$$

\section{Type-2 fuzzy linear system}

This section presents a class of T2FLS in which the coefficients are real crisp numbers and the variables are the triangular perfect QT2FNs. An approach, similar to what was proposed in Friedman et al. (1998), is introduced for obtaining the solution of T2FLS. In order for ideas to flow better and easier to follow the notations used in this section are tried to be compatible with that used in Friedman et al. (1998). Moreover, a definition of the type-2 fuzzy solution is presented.

A system of linear equations as $\left\{\begin{array}{l}a_{11} \tilde{x}_{1}+a_{12} \tilde{x}_{2}+\cdots+a_{1 n} \tilde{x}_{n}=\tilde{y}_{1} \\ a_{21} \tilde{x}_{1}+a_{22} \tilde{x}_{2}+\cdots+a_{2 n} \tilde{x}_{n}=\tilde{y}_{2} \\ \vdots \\ a_{n 1} \tilde{x}_{1}+a_{n 2} \tilde{x}_{2}+\cdots+a_{n n} \tilde{x}_{n}=\tilde{y}_{n}\end{array}\right.$

where $a_{i j} \in \mathbb{R}, 1 \leq i, j \leq n$ and $\tilde{x}_{i}, \tilde{y}_{i} \in E_{2}$ is called a T2FLS. T2FLS shown in Eq. (5) is expressed in the matrix form as $A \tilde{X}=\tilde{Y}$ in which

$A=\left[a_{i j}\right]_{n \times n}, \quad \tilde{X}=\left[\begin{array}{llll}\tilde{x}_{1} & \tilde{x}_{2} & \cdots & \tilde{x}_{n}\end{array}\right]^{T}$

and

$\tilde{Y}=\left[\begin{array}{llll}\tilde{y}_{1} & \tilde{y}_{2} & \cdots & \tilde{y}_{n}\end{array}\right]^{T}$.

According to Definition 9, we have

$\left(\underline{x}_{i \tilde{\alpha}}^{\alpha}, \bar{x}_{i \tilde{\alpha}}^{\alpha}\right)=\left(\left[\underline{L}_{x_{i \tilde{\alpha}}}^{\alpha}, \underline{R}_{i \tilde{\alpha}}^{\alpha}\right],\left[\bar{L}_{x_{i \tilde{\alpha}}}^{\alpha}, \bar{R}_{x_{i \tilde{\alpha}}}^{\alpha}\right]\right)$

and

$\left(\underline{y}_{i \tilde{\alpha}}^{\alpha}, \bar{y}_{i \tilde{\alpha}}^{\alpha}\right)=\left(\left[\underline{L}_{i \tilde{\alpha}}^{\alpha}, \underline{R}_{i \tilde{\alpha}}^{\alpha}\right],\left[\bar{L}_{y_{i \tilde{\alpha}}}^{\alpha}, \bar{R}_{y_{i \tilde{\alpha}}}^{\alpha}\right]\right)$.

Based on Theorem 2, solving T2FLS shown in Eq. (5) is equivalent to obtaining a solution of the following equations system:

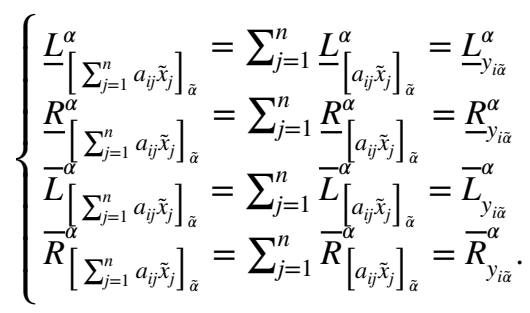

The system given by equations shown in Eq. (6) is a $4 n \times 4 n$ crisp linear system, where the right-hand side column is the $4 n \times 1$ matrix:

$\left[\begin{array}{lllllllll}\underline{L}_{y_{1 \tilde{\alpha}}}^{\alpha} & \cdots & \underline{L}_{y_{n \tilde{\alpha}}}^{\alpha} & R_{y_{1 \tilde{\alpha}}}^{\alpha} & \cdots & \underline{R}_{y_{n \tilde{\alpha}}}^{\alpha} & \bar{L}_{y_{1 \tilde{\alpha}}}^{\alpha} & \cdots & \bar{L}_{y_{n \tilde{\alpha}}}^{\alpha} \bar{R}_{y_{1 \tilde{\alpha}}}^{\alpha}\end{array}\right.$ $\left.\ldots \bar{R}_{y_{n \tilde{\alpha}}}^{\alpha}\right]^{T}$.

System shown in Eq. (6) can be rewritten in order that the $4 n \times 1$ matrix

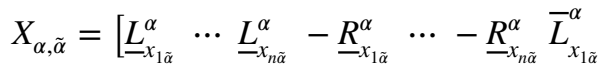

$$
\begin{aligned}
& \left.\cdots \bar{L}_{x_{n \tilde{\alpha}}}^{\alpha}-\bar{R}_{x_{1 \tilde{\alpha}}}^{\alpha} \ldots-\bar{R}_{x_{n \tilde{\alpha}}}^{\alpha}\right]^{T}
\end{aligned}
$$

includes the unknown variables, and the $4 n \times 1$ matrix, including the known variables in the right-hand side, is

$$
\begin{aligned}
& Y_{\alpha, \tilde{\alpha}}=\left[\begin{array}{lll}
\underline{L}_{y_{1 \tilde{\alpha}}}^{\alpha} & \cdots & \underline{L}_{y_{n \tilde{\alpha}}}^{\alpha}-\underline{R}_{y_{1 \tilde{\alpha}}}^{\alpha} \cdots-\underline{R}_{y_{n \tilde{\alpha}}}^{\alpha}
\end{array}\right. \\
& \left.\bar{L}_{y_{1 \tilde{\alpha}}}^{\alpha} \cdots \bar{L}_{y_{n \tilde{\alpha}}}^{\alpha}-\bar{R}_{y_{1 \tilde{\alpha}}^{\alpha}}^{\alpha} \cdots-\bar{R}_{y_{n \tilde{\alpha}}}^{\alpha}\right]^{T} .
\end{aligned}
$$

This rearranging leads to the system $S X_{\alpha, \tilde{\alpha}}=Y_{\alpha, \tilde{\alpha}}$, where $S$ is a real $4 n \times 4 n$ square matrix in the form of 


$$
S=\left[\begin{array}{llll}
B & C & \mathbf{0} & \mathbf{0} \\
C & B & \mathbf{0} & \mathbf{0} \\
\mathbf{0} & \mathbf{0} & B & C \\
\mathbf{0} & \mathbf{0} & C & B
\end{array}\right]
$$

in which $\mathbf{0}$ denotes an $n \times n$ zero matrix and the matrices $B=\left[b_{i j}\right]_{n \times n}, C=\left[c_{i j}\right]_{n \times n}$, are non-negative matrices, whose entries are determined as

$b_{i j}=\left\{\begin{array}{ll}a_{i j} & a_{i j}>0 \\ 0 & a_{i j} \leq 0\end{array} c_{i j}= \begin{cases}-a_{i j} & a_{i j}<0 \\ 0 & a_{i j} \geq 0 .\end{cases}\right.$

It is easy to see that $A=B-C$. It should be noted; if system shown in Eq. (6) does not have a unique solution, then T2FLS shown in Eq. (5) does not have a unique solution either. The unique solution to system shown in Eq. (6) can be found if and only if the matrix $S$ is invertible.

Theorem 3 The matrixSis invertible if and only if the matrices $A$ and $B+C$ are both invertible.

Proof It is similar to the proof of Theorem 1 in (Friedman et al. 1998).

Based on Theorem 3 presented in Friedman et al. (1998), a special case of T1FLSs has a unique fuzzy solution. What follows is a corollary of the Theorem 3 in the context of T2FLSs.

Corollary 1 T2FLS shown in Eq. (5) has a type-2 fuzzy solution, belonging to the triangular perfect QT2FNs, providing that matrix $S$ has an inverse matrix, $S^{-1}$, whose entries are non-negative.

Proof For the proof of this theorem, what is needed is to prove that $\bar{L}_{x_{i \tilde{\alpha}}}^{\alpha} \leq \underline{L}_{x_{i \tilde{\alpha}}}^{\alpha} \leq \underline{R}_{x_{i \tilde{\alpha}}}^{\alpha} \leq \bar{R}_{x_{i \tilde{\alpha}}^{\alpha}}^{\alpha}$. It is straightforward and hence omitted.

Although the conditions in Corollary 1 are sufficient conditions, and assure us that the unique type-2 fuzzy solution can be obtained, there is scarcely any matrix $S$ which satisfies the conditions. As a result, the following definition of solution is given.

Definition 12 Suppose $S^{-1}$ is the inverse of matrix $S$, and $Z_{\alpha, \tilde{\alpha}}=\left[z_{l}(\alpha, \tilde{\alpha})\right]_{4 n \times 1}, \quad l=1,2, \ldots, 4 n$, is a solution of $S Z_{\alpha, \tilde{\alpha}}=Y_{\alpha, \tilde{\alpha}}$, i.e., $Z_{\alpha, \tilde{\alpha}}=S^{-1} Y_{\alpha, \tilde{\alpha}}$. We say that $\tilde{X}=\left[\begin{array}{llll}\tilde{x}_{1} & \tilde{x}_{2} & \cdots & \tilde{x}_{n}\end{array}\right]^{T}$ is the type-2 fuzzy solution of T2FLS shown in Eq. (5) provided that

$\tilde{x}_{i}=\bigcup_{\tilde{\alpha} \in[0,1]} \tilde{\alpha} \bigcup_{\alpha \in[0,1]} \alpha \tilde{x}_{i \tilde{\alpha}}^{\alpha} \quad$ for all $i=1,2, \ldots, n$ represents the triangular perfect QT2FN, where $\tilde{x}_{i \tilde{\alpha}}^{\alpha}=\left(\underline{x}_{i \tilde{\alpha}}^{\alpha}, \bar{x}_{i \tilde{\alpha}}^{\alpha}\right)=\left(\left[\underline{L}_{x_{i \tilde{\alpha}}}^{\alpha}, \underline{R}_{x_{i \tilde{\alpha}}}^{\alpha}\right],\left[\bar{L}_{x_{i \tilde{\alpha}}}^{\alpha}, \bar{R}_{x_{i \tilde{\alpha}}}^{\alpha}\right]\right)$,

and

$\left\{\begin{array}{l}\underline{L}_{x_{i \tilde{\alpha}}}^{\alpha}=z_{i}(\alpha, \tilde{\alpha}) \\ -\underline{R}_{x_{i \tilde{\alpha}}}^{\alpha}=z_{i+n}(\alpha, \tilde{\alpha}) \\ \bar{L}_{x_{i \tilde{\alpha}}}^{\alpha}=z_{i+2 n}(\alpha, \tilde{\alpha}) \\ -\bar{R}_{x_{i \tilde{\alpha}}}^{\alpha}=z_{i+3 n}(\alpha, \tilde{\alpha}) .\end{array}\right.$

Based on the aforementioned, the following steps can be considered for obtaining the unique fuzzy solution of T2FLS shown in Eq. (5).

Step 1 Set up the matrices $Y_{\alpha, \tilde{\alpha}}$ and $S$ according to the relations shown in Eqs. (7), (8), and (9), respectively.

Step 2 Check whether the matrix $S$ is invertible or not. If the determinant of the matrix $S$ is nonzero, i.e., $|S| \neq 0$, then $S^{-1}$ exists, and go to the next step. If the determinant of the matrix $S$ is zero, i.e., $|S|=0$, then $S^{-1}$ does not exist, and therefore, the problem does not have a unique fuzzy solution.

Step 3 Obtain $Z_{\alpha, \tilde{\alpha}}=\left[z_{l}(\alpha, \tilde{\alpha})\right]_{4 n \times 1}, l=1,2, \ldots, 4 n$ using $Z_{\alpha, \tilde{\alpha}}=S^{-1} Y_{\alpha, \tilde{\alpha}}$.

Step 4 Using Eq. (10), determine $\underline{L}_{x_{i \tilde{\alpha}}}^{\alpha}, \underline{R}_{x_{i \tilde{\alpha}}}^{\alpha}, \bar{L}_{x_{i \tilde{\alpha}}}^{\alpha}$, and $\bar{R}_{x_{i \tilde{\alpha}}}^{\alpha}$ for each $\tilde{x}_{i \tilde{\alpha}}^{\alpha}$.

Step 5 Check whether $\quad\left(\left[\underline{L}_{x_{i \tilde{\alpha}}}^{\alpha}, \underline{R}_{x_{i \tilde{\alpha}}}^{\alpha}\right],\left[\bar{L}_{x_{i \tilde{\alpha}}}^{\alpha}, \bar{R}_{x_{i \tilde{\alpha}}}^{\alpha}\right]\right)$, $i=1,2, \ldots, n$ represents a $\mathrm{T} 2 \mathrm{FN}$ or not. To do that, we need to check whether the LMF, i.e., $\left[\underline{L}_{x_{i \tilde{\alpha}}}^{\alpha}, \underline{R}_{x_{i \tilde{\alpha}}}^{\alpha}\right]$, and UMF, i.e., $\left[\bar{L}_{x_{i \tilde{\alpha}}}^{\alpha}, \bar{R}_{x_{i \tilde{\alpha}}}^{\alpha}\right]$, are T1FNs them selves or not. If the LMF and UMF are T1FNs for each $i=1,2, \ldots, n$ and $\bar{L}_{w_{\tilde{\alpha}}}^{\alpha} \leq \underline{L}_{w_{\tilde{\alpha}}}^{\alpha} \leq \underline{R}_{w_{\tilde{\alpha}}}^{\alpha} \leq \bar{R}_{w_{\tilde{\alpha}}}^{\alpha}$, then according to Definition 10

$\tilde{x}_{i \tilde{\alpha}}^{\alpha}=\left(\underline{x}_{i \tilde{\alpha}}^{\alpha}, \bar{x}_{i \tilde{\alpha}}^{\alpha}\right)=\left(\left[\underline{L}_{x_{i \tilde{\alpha}}}^{\alpha}, \underline{R}_{x_{i \tilde{\alpha}}}^{\alpha}\right],\left[\bar{L}_{x_{i \tilde{\alpha}}}^{\alpha}, \bar{R}_{x_{i \tilde{\alpha}}}^{\alpha}\right]\right)$

represents a T2FN. Moreover, based on Definition 12, $\tilde{x}_{i \tilde{\alpha}}^{\alpha}=\left(\underline{x}_{i \tilde{\alpha}}^{\alpha}, \bar{x}_{i \tilde{\alpha}}^{\alpha}\right)$ is the unique fuzzy solution of T2FLS shown in Eq. (5) which can be characterized in the form of the septuple shown in Eq. (1).

Step 6 The unique fuzzy solution of T2FLS shown in Eq. (5) can be represented in the septuple form shown in Eq. (1) as

$\tilde{x}_{i}=\left(\bar{L}_{x_{i 0}}, L_{x_{i 1}}, \underline{L}_{x_{i 0}}, m, \underline{R}_{x_{i 0}}, R_{x_{i 1}}, \bar{R}_{x_{i 0}}\right)$

where based on the relations shown in Eqs. (2), (3), and (4)

$\bar{L}_{x_{i 0}}=\left.\bar{L}_{x_{i \tilde{\alpha}}}^{\alpha}\right|_{\tilde{\alpha}, \alpha=0}, \quad L_{x_{i 1}}=\left.\bar{L}_{x_{i \tilde{\alpha}}}^{\alpha}\right|_{\tilde{\alpha}=1, \alpha=0}$,

$\underline{L}_{x_{i 0}}=\left.\underline{L}_{x_{i \tilde{\alpha}}}^{\alpha}\right|_{\tilde{\alpha}, \alpha=0}, \quad m=\left.\underline{L}_{x_{i \tilde{\alpha}}}^{\alpha}\right|_{\tilde{\alpha}, \alpha=1}$,

$\bar{R}_{x_{i 0}}=\left.\bar{R}_{x_{i \tilde{\alpha}}}^{\alpha}\right|_{\tilde{\alpha}, \alpha=0}, \quad R_{x_{i 1}}=\left.\bar{R}_{x_{i \tilde{\alpha}}}^{\alpha}\right|_{\tilde{\alpha}=1, \alpha=0}$,

$\underline{R}_{x_{i 0}}=\left.\underline{R}_{x_{i \tilde{\alpha}}}^{\alpha}\right|_{\tilde{\alpha}, \alpha=0}$. 


\section{Examples}

Example 1 A stage in the retrofit strategy for the reduction of water and energy in pulp and paper processes is considered as an application of type- 2 fuzzy linear system. The stage corresponds to pulp washing process. Pulp and paper are manufactured from raw materials containing cellulose fibers, generally wood, recycled paper, and agricultural residues (Bajpai 2012). The process of pulp washing has been shown in Fig. 3 which has been adopted from (Patino and Nunez 1998).

In this case, we are going to obtain the values of the pulp flowing out from filter 1,2 and the wastewater from filter 2. The unknowns are concentrations. The information has been obtained by the aim of the experiences of an expert and others with a level lower than that of the expert. Depending on their experience and expertise, the experts mention different uncertainty for the concentration values of pulp flowing into the blow tank, wastewater flowing out filter 1 , and pulp flowing into filter 3 . If it is not possible to present an exact form of a type-1 fuzzy number for each of the values, in which the specialized experience of all the experts with different levels of expertise is reflected hierarchically, then type- 2 fuzzy numbers may prove helpful in this case. This could be considered in the way that the more reliable an expert's experience, compared to all of the others', the higher $\tilde{\alpha}$-plane includes his/her membership functions. Using the mass balance for each species, the following equations are obtained:

$$
\left[\begin{array}{lll}
280 & 30 & 50 \\
0 & 30 & 50 \\
280 & 0 & 50
\end{array}\right]\left[\begin{array}{l}
\tilde{c}_{6} \\
\tilde{c}_{8} \\
\tilde{c}_{9}
\end{array}\right]=\left[\begin{array}{ll}
83 & \tilde{c}_{1} \\
65 & \tilde{c}_{5} \\
785 & \tilde{c}_{12}
\end{array}\right]
$$

where

$$
\left\{\begin{array}{l}
\tilde{c}_{1}=(5,7,8,10,12,13,15), \\
\tilde{c}_{5}=(1.5,1.7,1.8,2,2.2,2.3,2.5), \\
\tilde{c}_{12}=(0.5,0.7,0.8,1,1.2,1.3,1.5) .
\end{array}\right.
$$

According to the steps expressed in the previous section, we have:

Step 1 According to the relation shown in Eq. (7), we have

$$
\begin{aligned}
Y_{\alpha, \tilde{\alpha}}= & {\left[83 \underline{L}_{c_{1 \tilde{\alpha}}}^{\alpha} 65 \underline{L}_{c_{5 \tilde{\alpha}}}^{\alpha} 785 \underline{L}_{c_{12 \tilde{\alpha}}}^{\alpha}-83 \underline{R}_{c_{1 \tilde{\alpha}}}^{\alpha}-65 \underline{R}_{c_{5 \tilde{\alpha}}}^{\alpha}\right.} \\
& -785 \underline{R}_{c_{12 \tilde{\alpha}}^{\alpha}}^{\alpha} 83 \bar{L}_{c_{1 \tilde{\alpha}}^{\alpha}}^{\alpha} 65 \bar{L}_{c_{5 \tilde{\alpha}}}^{\alpha} 785 \bar{L}_{c_{12 \tilde{\alpha}}}^{\alpha}-83 \bar{R}_{c_{1 \tilde{\alpha}}}^{\alpha} \\
& \left.-65 \bar{R}_{c_{5 \tilde{\alpha}}}^{\alpha}-785 \bar{R}_{c_{12 \tilde{\alpha}}}^{\alpha}\right]^{T}
\end{aligned}
$$

where based on the relations shown in Eqs. (2), (3), and (4)

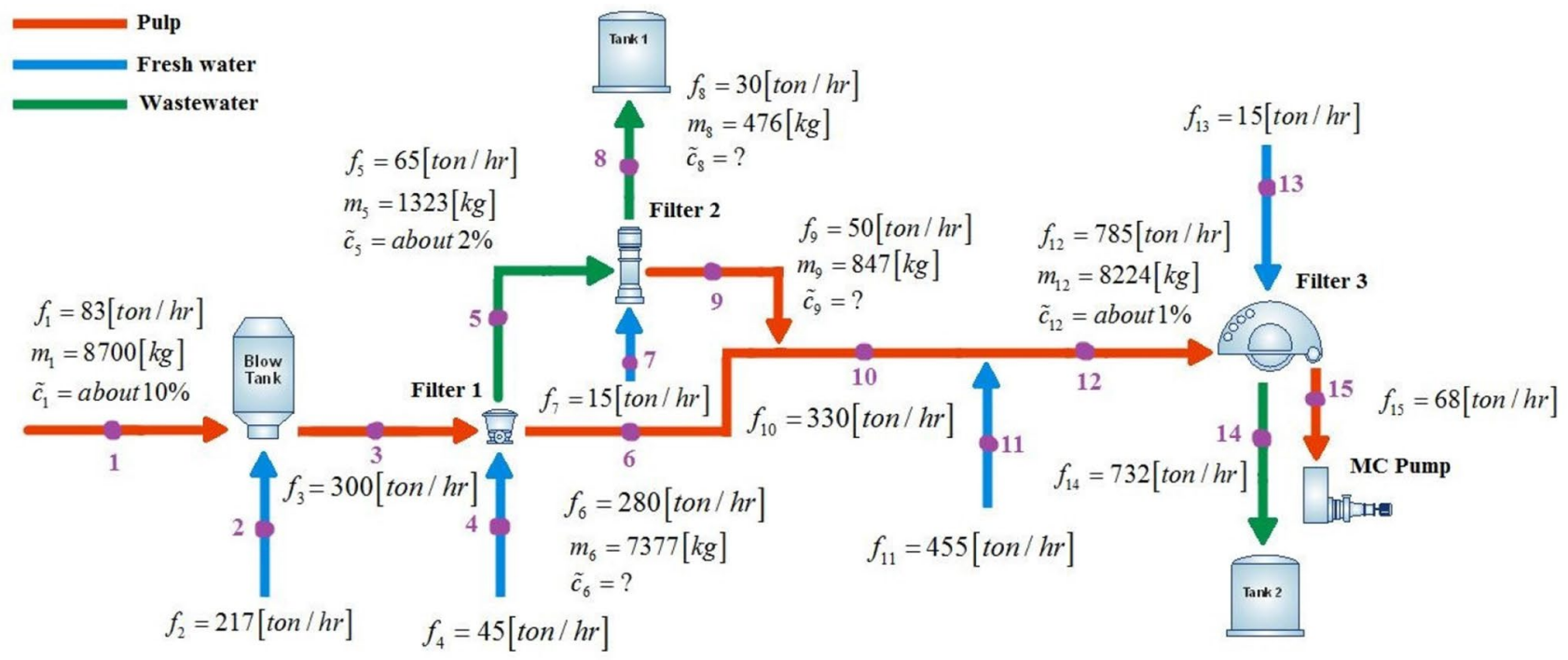

Fig. 3 Process of pulp washing. Originally published in Patino and Nunez (1998) under CC BY-NC-SA 3.0 license. Available from: http:// dx.doi.org/10.5772/20882 
$\underline{L}_{c_{1 \tilde{\alpha}}}^{\alpha}=7+3 \alpha-(1-\tilde{\alpha})(\alpha-1)$
$\underline{R}_{c_{1 \tilde{\alpha}}}^{\alpha}=13-3 \alpha-(1-\tilde{\alpha})(1-\alpha)$
$\bar{L}_{c_{1 \tilde{\alpha}}}^{\alpha}=7+3 \alpha-(1-\tilde{\alpha})(2-2 \alpha)$
$\bar{R}_{c_{1 \tilde{\alpha}}}^{\alpha^{\alpha}}=13-3 \alpha-(1-\tilde{\alpha})(2 \alpha-2)$
$\underline{L}_{c_{5 \tilde{\alpha}}}^{\alpha}=1.7+0.3 \alpha-(1-\tilde{\alpha})(0.1 \alpha-0.1)$
$\underline{R}_{\bar{L}_{5 \tilde{\alpha}}}^{\alpha}=2.3-0.3 \alpha-(1-\tilde{\alpha})(0.1-0.1 \alpha)$
$\bar{L}_{c_{5 \tilde{}}}^{\alpha}=1.7+0.3 \alpha-(1-\tilde{\alpha})(0.2-0.2 \alpha)$
$\bar{R}_{c_{5 \tilde{\alpha}}}^{\alpha^{\alpha}}=2.3-0.3 \alpha-(1-\tilde{\alpha})(0.2 \alpha-0.2)$
$\underline{L}_{c_{12 \tilde{\alpha}}}^{\alpha}=0.7+0.3 \alpha-(1-\tilde{\alpha})(0.1 \alpha-0.1)$
$\bar{R}_{c_{12 \tilde{\alpha}}}^{\alpha}=1.3-0.3 \alpha-(1-\tilde{\alpha})(0.1-0.1 \alpha)$
$\bar{L}_{c_{12 \tilde{\alpha}}}^{\alpha}=0.7+0.3 \alpha-(1-\tilde{\alpha})(0.2-0.2 \alpha)$
$\bar{R}_{c_{12 \tilde{\alpha}}}^{\alpha^{2}}=1.3-0.3 \alpha-(1-\tilde{\alpha})(0.2 \alpha-0.2)$

and by the use of the relations shown in Eqs. (8) and (9), we have

$$
S=\left[\begin{array}{llll}
B & \mathbf{0} & \mathbf{0} & \mathbf{0} \\
\mathbf{0} & B & \mathbf{0} & \mathbf{0} \\
\mathbf{0} & \mathbf{0} & B & \mathbf{0} \\
\mathbf{0} & \mathbf{0} & \mathbf{0} & B
\end{array}\right]
$$

in which

$$
B=\left[\begin{array}{lll}
280 & 30 & 50 \\
0 & 30 & 50 \\
280 & 0 & 50
\end{array}\right] .
$$

Step 2 The determinant of the matrix $S$ is $|S|=|B|^{4} \neq 0$, then $S$ is invertible and we go to the next step.

Step 3 The matrix $Z_{\alpha, \tilde{\alpha}}=\left[z_{l}(\alpha, \tilde{\alpha})\right]_{12 \times 1}$, is obtained as

$z_{1}(\alpha, \tilde{\alpha})=1.68+0.82 \alpha-(1-\tilde{\alpha})(0.27 \alpha-0.27)$

$z_{2}(\alpha, \tilde{\alpha})=1.05+0.45 \alpha-(1-\tilde{\alpha})(0.15 \alpha-0.15)$

$z_{3}(\alpha, \tilde{\alpha})=1.58+0.12 \alpha-(1-\tilde{\alpha})(0.04 \alpha-0.04)$

$z_{4}(\alpha, \tilde{\alpha})=-3.32+0.82 \alpha-(1-\tilde{\alpha})(0.27 \alpha-0.27)$

$z_{5}(\alpha, \tilde{\alpha})=-1.95+0.45 \alpha-(1-\tilde{\alpha})(0.15 \alpha-0.15)$

$z_{6}(\alpha, \tilde{\alpha})=-1.82+0.12 \alpha-(1-\tilde{\alpha})(0.04 \alpha-0.04)$

$z_{7}(\alpha, \tilde{\alpha})=1.68+0.82 \alpha-(1-\tilde{\alpha})(0.55-0.55 \alpha)$

$z_{8}(\alpha, \tilde{\alpha})=1.05+0.45 \alpha-(1-\tilde{\alpha})(0.3-0.3 \alpha)$

$z_{9}(\alpha, \tilde{\alpha})=1.58+0.12 \alpha-(1-\tilde{\alpha})(0.08-0.08 \alpha)$

$z_{10}(\alpha, \tilde{\alpha})=-3.32+0.82 \alpha-(1-\tilde{\alpha})(0.55-0.55 \alpha)$

$z_{11}(\alpha, \tilde{\alpha})=-1.95+0.45 \alpha-(1-\tilde{\alpha})(0.3-0.3 \alpha)$

$z_{12}(\alpha, \tilde{\alpha})=-1.82+0.12 \alpha-(1-\tilde{\alpha})(0.08-0.08 \alpha)$.

Step 4 Using the relation shown in Eq. (10), we have
$\underline{L}_{c_{6 \tilde{\alpha}}}^{\alpha}=1.68+0.82 \alpha-(1-\tilde{\alpha})(0.27 \alpha-0.27)$

$\underline{R}_{c_{6 \tilde{\alpha}}}^{\alpha}=3.32-0.82 \alpha+(1-\tilde{\alpha})(0.27 \alpha-0.27)$

$\bar{L}_{c_{6 \tilde{\alpha}}}^{\alpha_{6 \tilde{\alpha}}}=1.68+0.82 \alpha-(1-\tilde{\alpha})(0.55-0.55 \alpha)$

$\bar{R}_{c_{6 \tilde{\alpha}}^{\alpha \tilde{\alpha}}}^{\alpha^{\delta}}=3.32-0.82 \alpha+(1-\tilde{\alpha})(0.55-0.55 \alpha)$

$\underline{L}_{c_{8 \tilde{\alpha}}^{\alpha}}^{\alpha_{6 \tilde{\alpha}}}=1.05+0.45 \alpha-(1-\tilde{\alpha})(0.15 \alpha-0.15)$

$\underline{R}_{c_{8 \tilde{\alpha}}}^{\alpha}=1.95-0.45 \alpha+(1-\tilde{\alpha})(0.15 \alpha-0.15)$

$\bar{L}_{c_{8 \tilde{\alpha}}}^{\alpha^{8 \tilde{\alpha}}}=1.05+0.45 \alpha-(1-\tilde{\alpha})(0.3-0.3 \alpha)$

$\bar{R}_{c_{8 \tilde{\alpha}}}^{c_{\delta \tilde{\alpha}}}=1.95-0.45 \alpha+(1-\tilde{\alpha})(0.3-0.3 \alpha)$

$\underline{L}_{c_{9 \tilde{\alpha}}}^{c_{8 \tilde{\alpha}}}=1.58+0.12 \alpha-(1-\tilde{\alpha})(0.04 \alpha-0.04)$

$\underline{R}_{C_{9 \tilde{\alpha}}}^{\alpha}=1.82-0.12 \alpha+(1-\tilde{\alpha})(0.04 \alpha-0.04)$

$\bar{L}_{c_{9 \tilde{\alpha}}}^{\alpha^{9 \tilde{\alpha}}}=1.58+0.12 \alpha-(1-\tilde{\alpha})(0.08-0.08 \alpha)$

$\frac{C_{c_{9 \tilde{\alpha}}}^{\alpha^{\alpha}}}{R_{c^{2}}}=1.82-0.12 \alpha+(1-\tilde{\alpha})(0.08-0.08 \alpha)$.

Step 5 It is easy to see that $\left[\underline{L}_{c_{6 \tilde{\alpha}}}^{\alpha}, \underline{R}_{c_{6 \tilde{\alpha}}}^{\alpha}\right]$ represents the $\alpha$-level sets of a T1FN for each $\tilde{\alpha} \in[0,1]$, and $\left[\bar{L}_{c_{6 \tilde{\alpha}}}^{\alpha}, \bar{R}_{c_{6 \tilde{\alpha}}}^{\alpha}\right]$ does too. In addition, since $\bar{L}_{c_{6 \tilde{\alpha}}}^{\alpha} \leq \underline{L}_{c_{6 \tilde{\alpha}}}^{\alpha} \leq \underline{R}_{c_{6 \tilde{\alpha}}}^{\alpha} \leq \bar{R}_{c_{6 \tilde{\alpha}}}^{\alpha}$, then

$\left[\tilde{c}_{6}\right]_{\tilde{\alpha}}^{\alpha}=\left(\left[\underline{L}_{c_{6 \tilde{\alpha}}}^{\alpha}, \underline{R}_{c_{6 \tilde{\alpha}}}^{\alpha}\right],\left[\bar{L}_{c_{6 \tilde{\alpha}}}^{\alpha}, \bar{R}_{c_{6 \tilde{\alpha}}}^{\alpha}\right]\right)$

represents a T2FN. In a similar way, it can be investigated that $\quad\left[\tilde{c}_{8}\right]_{\tilde{\alpha}}^{\alpha}=\left(\left[\underline{L}_{c_{8 \tilde{\alpha}}}^{\alpha}, \underline{R}_{c_{8 \tilde{\alpha}}}^{\alpha}\right],\left[\bar{L}_{c_{8 \tilde{\alpha}}^{\alpha}}^{\alpha}, \bar{R}_{c_{8 \tilde{\alpha}}^{\alpha}}^{\alpha}\right]\right) \quad$ and $\left[\tilde{c}_{9}\right]_{\tilde{\alpha}}^{\alpha}=\left(\left[\underline{L}_{c_{9 \tilde{\alpha}}}^{\alpha}, \underline{R}_{c_{9 \tilde{\alpha}}}^{\alpha}\right],\left[\bar{L}_{c_{9 \tilde{\alpha}}^{\alpha}}^{\alpha}, \bar{R}_{c_{9 \tilde{\alpha}}}^{\alpha}\right]\right)$ also represent two T2FNs.

Step 6 Consequently, the fuzzy solution of the system shown in Eq. (11) can be characterized in the septuple form as

$\left\{\begin{array}{l}\tilde{c}_{6}=(1.13,1.68,1.95,2.5,3.05,3.32,3.87), \\ \tilde{c}_{8}=(0.75,1.05,1.2,1.5,1.8,1.95,2.25), \\ \tilde{c}_{9}=(1.5,1.58,1.62,1.7,1.78,1.82,1.9) .\end{array}\right.$

As a result, based on the multiplication of a scalar by a T2FN defined in Sect. 3, the values of the pulp flowing out from filter 1, 2 and the wastewater from filter 2 are obtained by the multiplication of $f_{6}$ by $\tilde{c}_{6}, f_{8}$ by $\tilde{c}_{8}$ and $f_{9}$ by $\tilde{c}_{9}$, respectively, as

$f_{6} \tilde{c}_{6}=(316.4,470.4,546,700,854,929.6,1083.6)$,

$f_{8} \tilde{c}_{8}=(22.5,31.5,36,45,54,58.5,67.5)$,

and

$f_{9} \tilde{c}_{9}=(75,79,81,85,89,91,95)$

which mean approximately 700,45 , and 85 . It should be noted that using the obtained results, approximately 700 
Fig. 4 Electrical circuit considered in Example 2

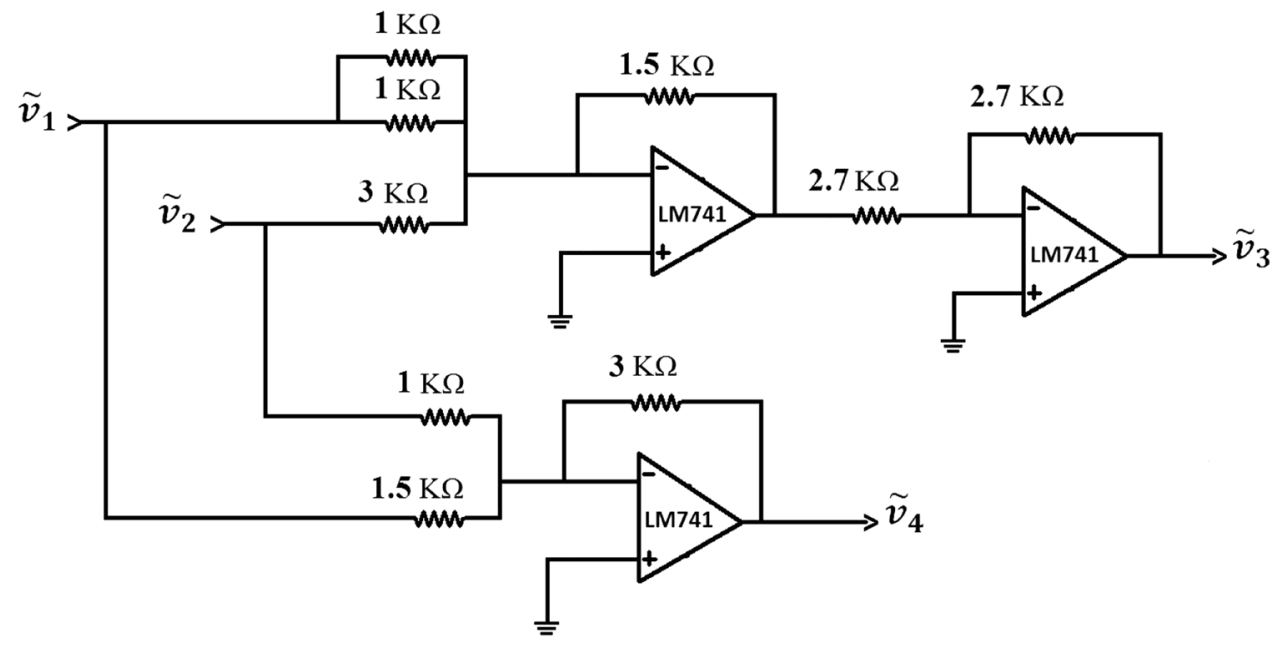

also means the T1FN $(470.4,700,929.6)$, whose uncertainty dispersion can be considered by its UMF and LMF as $(316.4,700,1083.6)$ and $(546,700,854)$, respectively. The uncertainty dispersion for approximately 45 and 85 can also be expressed in a similar way. Needless to pinpoint once more that, unlike T2FLSs, T1FLSs cannot provide any information about the uncertainty dispersion of the results.

Example 2 Consider the electrical circuit shown in Fig. 4, where $\tilde{v}_{1}$ and $\tilde{v}_{2}$ are the input voltages, and $\tilde{v}_{3}$ and $\tilde{v}_{4}$ are the output voltages. The circuit is a kind of summing amplifier with two inputs and two outputs. The relationship between input and output voltages is as follows: $\left[\begin{array}{ll}3 & 0.5 \\ -2 & -3\end{array}\right]\left[\begin{array}{l}\tilde{v}_{1} \\ \tilde{v}_{2}\end{array}\right]=\left[\begin{array}{l}\tilde{v}_{3} \\ \tilde{v}_{4}\end{array}\right]$

What we are investigating is determining the input voltages on condition that the output voltages are known but uncertain. That is, e.g., $\tilde{v}_{3}$ is "about 16 (volt)", and $\tilde{v}_{4}$ is "about -16 (volt)". Assume, the engineers' expert ideas about the uncertainty of output voltages differ. If we prefer to consider the interpretation of just one, not all of the experts, the linear system shown in Eq. (13) will be a T1FLS one, failing to consider the interpretation or experience of the other experts. Alternatively, we may consider a form of T1FN for each of the output voltages, based on the experiences of the experts that, in turn, leads to the presentation of T1FLS. Even this model of system fails to hierarchically reflect the specialized experience of all the experts
Fig. 5 Output voltage, $\tilde{v}_{3}$, corresponding to the electrical circuit

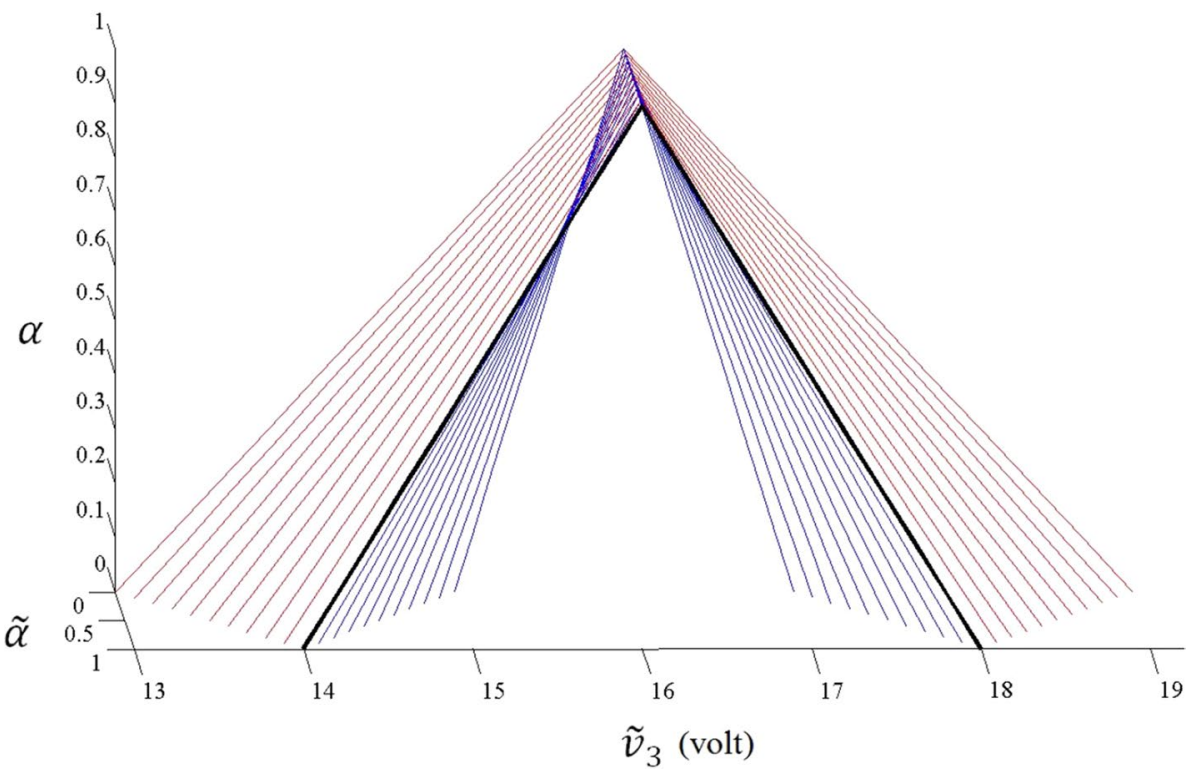


Fig. 6 Output voltage, $\tilde{v}_{4}$, corresponding to the electrical circuit

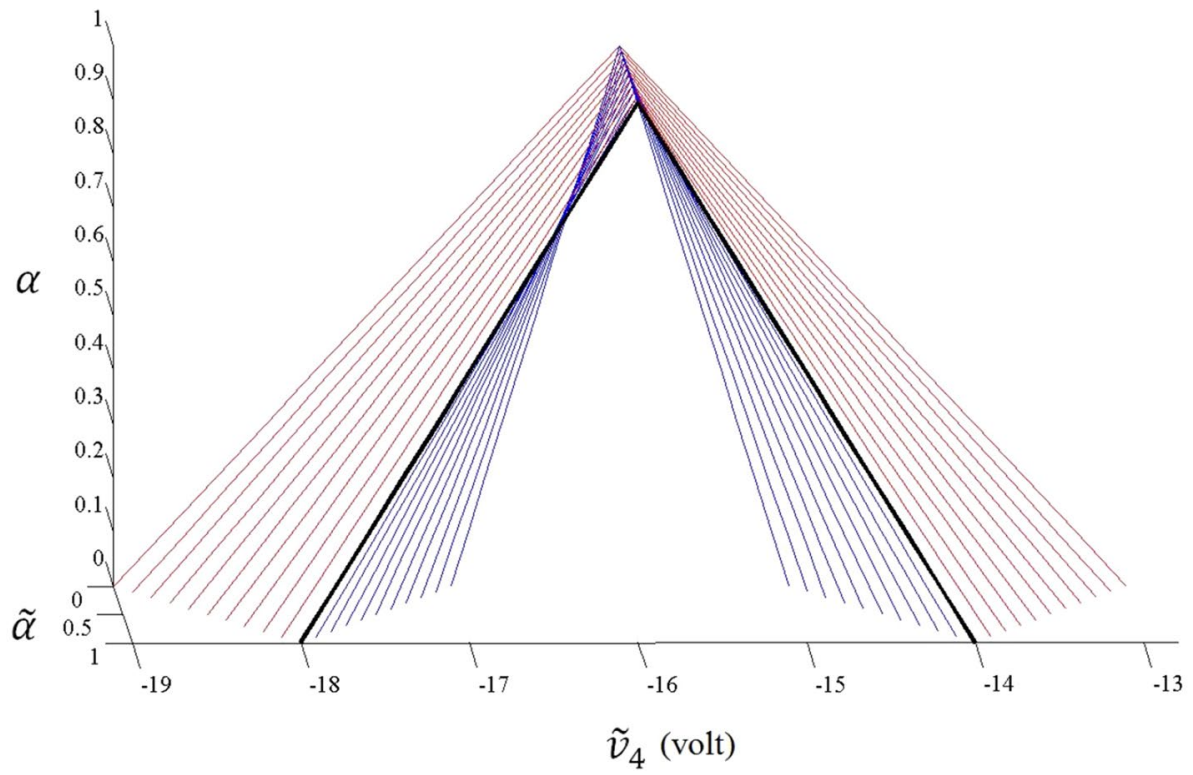

with different levels of expertise. However, each of the output voltages may be determined using a triangular perfect QT2FN, as shown in Figs. 5 and 6. For determining the input voltages, the matrix $Z_{\alpha, \tilde{\alpha}}=\left[z_{l}(\alpha, \tilde{\alpha})\right]_{8 \times 1}$ is needed to be obtained. Then, according to the steps expressed in the previous section, we have:

Step 1 The matrices $S$ and $Y_{\alpha, \tilde{\alpha}}$ can be gained according to the relations shown in Eqs. (7), (8), and (9), respectively, as follows:

$S=\left[\begin{array}{cccccccc}3 & 0.5 & 0 & 0 & 0 & 0 & 0 & 0 \\ 0 & 0 & -2 & -3 & 0 & 0 & 0 & 0 \\ 0 & 0 & 3 & 0.5 & 0 & 0 & 0 & 0 \\ -2 & -3 & 0 & 0 & 0 & 0 & 0 & 0 \\ 0 & 0 & 0 & 0 & 3 & 0.5 & 0 & 0 \\ 0 & 0 & 0 & 0 & 0 & 0 & -2 & -3 \\ 0 & 0 & 0 & 0 & 0 & 0 & 3 & 0.5 \\ 0 & 0 & 0 & 0 & -2 & -3 & 0 & 0\end{array}\right]$

$$
\begin{aligned}
Y_{\alpha, \tilde{\alpha}}= & {\left[\underline{L}_{v_{3 \tilde{\alpha}}}^{\alpha} \underline{L}_{v_{4 \tilde{\alpha}}}^{\alpha}-\underline{R}_{v_{3 \tilde{\alpha}}}^{\alpha}-\underline{R}_{v_{4 \tilde{\alpha}}}^{\alpha} \bar{L}_{v_{3 \tilde{\alpha}}^{\alpha}}^{\alpha}\right.} \\
& \left.\bar{L}_{v_{4 \tilde{\alpha}}}^{\alpha}-\bar{R}_{v_{3 \tilde{\alpha}}}^{\alpha}-\bar{R}_{v_{4 \tilde{\alpha}}}^{\alpha}\right]^{T}
\end{aligned}
$$

where based on the relations shown in Eqs. (2), (3), and (4)

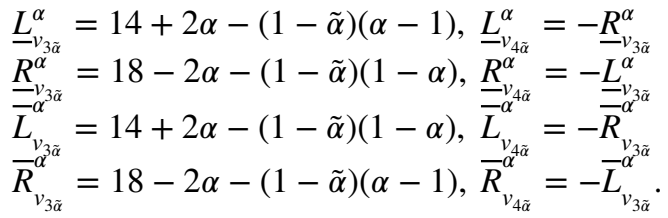

Step 2 The determinant of the matrix $S$ is $|S|=4096$, then the matrix $S$ is invertible and we go to the next step.
Step 3 The matrix $Z_{\alpha, \tilde{\alpha}}=\left[z_{l}(\alpha, \tilde{\alpha})\right]_{8 \times 1}$ is determined as

$z_{1}(\alpha, \tilde{\alpha})=4.37+0.63 \alpha-(1-\tilde{\alpha})(0.32 \alpha-0.32)$

$z_{2}(\alpha, \tilde{\alpha})=1.75+0.25 \alpha-(1-\tilde{\alpha})(0.13 \alpha-0.13)$

$z_{3}(\alpha, \tilde{\alpha})=-5.63+0.63 \alpha-(1-\tilde{\alpha})(0.32 \alpha-0.32)$

$z_{4}(\alpha, \tilde{\alpha})=-2.25+0.25 \alpha-(1-\tilde{\alpha})(0.13 \alpha-0.13)$

$z_{5}(\alpha, \tilde{\alpha})=4.37+0.63 \alpha-(1-\tilde{\alpha})(0.31-0.31 \alpha)$

$z_{6}(\alpha, \tilde{\alpha})=1.75+0.25 \alpha-(1-\tilde{\alpha})(0.12-0.12 \alpha)$

$z_{7}(\alpha, \tilde{\alpha})=-5.63+0.63 \alpha-(1-\tilde{\alpha})(0.31-0.31 \alpha)$

$z_{8}(\alpha, \tilde{\alpha})=-2.25+0.25 \alpha-(1-\tilde{\alpha})(0.12-0.12 \alpha)$

Step 4 Using the relation shown in Eq. (10), we have

$\underline{L}_{v_{1 \tilde{\alpha}}}^{\alpha}=4.37+0.63 \alpha-(1-\tilde{\alpha})(0.32 \alpha-0.32)$,

$\underline{R}_{v_{1 \tilde{\alpha}}}^{\alpha}=5.63-0.63 \alpha+(1-\tilde{\alpha})(0.32 \alpha-0.32)$,

$\bar{L}_{v_{1 \tilde{\alpha}}}^{\alpha_{1 \tilde{\alpha}}}=4.37+0.63 \alpha-(1-\tilde{\alpha})(0.31-0.31 \alpha)$,

$\bar{R}_{v_{1 \tilde{\alpha}}}^{\alpha_{1 \tilde{\alpha}}}=5.63-0.63 \alpha+(1-\tilde{\alpha})(0.31-0.31 \alpha)$,

$\underline{L}_{v_{2 \alpha}}^{\alpha}=1.75+0.25 \alpha-(1-\tilde{\alpha})(0.13 \alpha-0.13)$

$\underline{R}_{v_{2 \tilde{\alpha}}}^{\alpha}=2.25-0.25 \alpha+(1-\tilde{\alpha})(0.13 \alpha-0.13)$

$\overline{\bar{L}}_{v_{2 \tilde{\alpha}}}^{\alpha_{2 \tilde{\alpha}}}=1.75+0.25 \alpha-(1-\tilde{\alpha})(0.12-0.12 \alpha)$

$\bar{R}_{v_{2 \tilde{\alpha}}}^{\alpha^{2 \tilde{\alpha}}}=2.25-0.25 \alpha+(1-\tilde{\alpha})(0.12-0.12 \alpha)$.

Step 5 It is easy to see that $\left[\underline{L}_{v_{1 \tilde{\alpha}}}^{\alpha}, \underline{R}_{v_{1 \tilde{\alpha}}}^{\alpha}\right]$ represents the $\alpha$-level sets of a T1FN for each $\tilde{\alpha} \in[0,1]$, and $\left[\bar{L}_{v_{1 \tilde{\alpha}}}^{\alpha}, \bar{R}_{v_{1 \tilde{\alpha}}}^{\alpha}\right]$ does too. In addition, since $\bar{L}_{v_{1 \tilde{\alpha}}}^{\alpha} \leq \underline{L}_{v_{1 \tilde{\alpha}}}^{\alpha} \leq \underline{R}_{v_{1 \tilde{\alpha}}}^{\alpha} \leq \bar{R}_{v_{1 \tilde{\alpha}}}^{\alpha}$, then

$\left[\tilde{v}_{1}\right]_{\tilde{\alpha}}^{\alpha}=\left(\left[\underline{L}_{v_{1 \tilde{\alpha}}}^{\alpha}, \underline{R}_{v_{1 \tilde{\alpha}}}^{\alpha}\right],\left[\bar{L}_{v_{1 \tilde{\alpha}}}^{\alpha}, \bar{R}_{v_{1 \tilde{\alpha}}}^{\alpha}\right]\right)$

represents a T2FN. In a similar way, it can be investigated that $\left[\tilde{v}_{2}\right]_{\tilde{\alpha}}^{\alpha}=\left(\left[\underline{L}_{v_{2 \tilde{\alpha}}}^{\alpha}, \underline{R}_{v_{2 \tilde{\alpha}}}^{\alpha}\right],\left[\bar{L}_{v_{2 \tilde{\alpha}}}^{\alpha}, \bar{R}_{v_{2 \tilde{\alpha}}}^{\alpha}\right]\right)$ also represents a T2FN. 
Step 6 Eventually, the input voltages can be expressed as

$\tilde{v}_{1}=(4.06,4.37,4.69,5,5.31,5.63,5.94)$,

$\tilde{v}_{2}=(1.63,1.75,1.88,2,2.12,2.25,2.37)$.

The input voltages $\tilde{v}_{1}$ and $\tilde{v}_{2}$ are two T2FNs, whose cores are 5 and 2, respectively. Due to uncertainty, they are not exactly 5 and 2 , but they can be interpreted as approximately 5 and approximately 2 . As a result, one can interpret that the circuit amplifies approximately 5 and 2 (volt) to approximately 16 and -16 (volt), respectively.

\section{Conclusion}

In this paper, a model of fuzzy linear system called T2FLS has been established that enables us to model linear systems in which in addition to data, the membership function itself is uncertain. T2FLS, unlike T1FLS, provides information about uncertainty dispersion. It was shown that how an $n \times n$ T2FLS is replaced by a $4 n \times 4 n$ crisp linear system. Furthermore, the conditions for the existence of a unique type- 2 fuzzy solution to the $n \times n$ T2FLS were given. Since the conditions may not always occur, a definition of type-2 fuzzy solution has been defined. Using two examples in the pulp and paper industry and electrical engineering, we showed that how the T2FLSs enable the decision maker to model the system with different interpretations of uncertainty expressed by experts in different levels. Due to the fact that T2FLSs with the help of T2FNs model higher levels uncertainty than those do the T1FLSs, this opens up an efficient way for modeling linear system and human decision making. We believe that what have been presented in this paper can be used to extend many existing T1FLSs result to T2FLSs. The next step in the research direction proposed here is to investigate T2FLSs in which all the variables and coefficients are T2FNs.

\section{References}

Abbasbandy S, Ezzati R, Jafarian A (2006) LU decomposition method for solving fuzzy system of linear equations. Appl Math Comput 172:633-643

Abbasbandy S, Jafarian A (2006) Steepest descent method for system of fuzzy linear equations. Appl Math Comp 175:823-833

Bajpai P (2012) Biotechnol Pulp Paper Processing. Springer, US

Behera D, Chakraverty S (2014) Solving fuzzy complex system of linear equations. Inform Sci 277:154-162

Buckley JJ, Qu Y (1991) Solving systems of linear fuzzy equations. Fuzzy Sets Syst 43:33-43
Coupland S, John RI (2007) Geometric Type-1 and Type-2 Fuzzy Logic Systems. IEEE Trans Fuzzy Syst 15:3-15

Das S, Kar S, Pal T (2017) Robust decision making using intuitionistic fuzzy numbers. Granul Comput. doi:10.1007/ s41066-016-0024-3

Friedman M, Ming M, Kandel A (1998) Fuzzy linear systems. Fuzzy Sets Syst 96:201-209

Ghanbari R (2015) Solutions of fuzzy LR algebraic linear systemsusing linear programs. Appl Math Model 39:5164-5173

Garibaldi JM, Zhou SM, Wang XY, John RI, Ellis IO (2012) Incorporation of Expert Variability into Breast Cancer Treatment Recommendation in Designing Clinical Protocol Guided Fuzzy Rule System Models. J Biomed Inform 45:447-459

Hamrawi H, Coupland S, John RI (2010) A novel alpha-cut representation for type-2 fuzzy sets. In: IEEE international conference on fuzzy systems, Barcelona, Spain. doi:10.1109/ FUZZY.2010.5584783

Hamrawi H (2011) Type-2 fuzzy alpha-cuts. Ph.D. Dissertation, De Montfort University

Hamrawi H, Coupland S, John RI (2016) Type-2 fuzzy alpha-cuts. IEEE Trans Fuzzy Syst. doi:10.1109/TFUZZ.2016.2574914

Hung WL, Yang MS (2004) Similarity measures between type-2 fuzzy sets. Int J Uncertain Fuzziness Knowl Based Syst $12: 827-841$

John RI, Innocent PR (2005) Modeling uncertainty in clinical diagnosis using fuzzy logic. IEEE Trans Syst Man Cybern Part B: Cybern 35:1340-1350

Liu F (2008) An efficient centroid type-reduction strategy for general type-2 fuzzy logic system. Inform Sci 178:2224-2236

Mendel JM (2007) Type-2 fuzzy sets and systems: an overview. IEEE Comput Intell Mag 2:20-9

Mendel JM, Liu F (2008) On new quasi-type-2 fuzzy logic systems. Proceeding of 2008 international conference on fuzzy systems, Proceedings of the 2008 IEEE International Conference on Fuzzy Systems. Hong Kong, China, pp 354-360

Mendel JM (2016) A comparison of three approaches for estimating (synthesizing) an interval type-2 fuzzy set model of a linguistic term for computing with words. Granul Comput 1:59-69

Ming M, Friedman M, Kandel A (2000) Duality in fuzzy linear systems. Fuzzy Sets Syst 109:55-58

Mendel JM, John RI (2002) Type-2 fuzzy sets made simple. IEEE Trans Fuzzy Syst 10:117-127

Mazandarani M, Kamyad AV (2011) A practical approach to prescribe the amount of used insulin of diabetic patients. 19th Iranian Conference on Electrical Engineering, Tehran, Iran, pp. 3158-3162

Mendel JM, Liu F, Zhai D (2009) $\alpha$-Plane representation for type-2 fuzzy sets: theory and applications. IEEE Trans Fuzzy Syst 17:1189-207

Mazandarani M, Najariyan M (2014) Type-2 fuzzy fractional derivatives. Commun Nonlinear Sci Numer Simulat 19:2354-2372

Mazandarani M, Najariyan M (2014) Differentiability of type-2 fuzzy number-valued Functions. Commun Nonlinear Sci Numer Simul 19:710-25

Muzzioli S, Reynaerts H (2006) fuzzy linear systems of the form $A_{1} x+b_{1}=A_{2} X+b_{2}$. Fuzzy Sets Syst 157:939-951

Najariyan M, Farahi MH, Alavian M (2011) Optimal control of HIV infection by using fuzzy dynamical systems. J Math Comput Sci 2:639-49

Najariyan M, Farahi MH (2015) A new approach for solving a class of fuzzy optimal control systems under generalized Hukuhara differentiability. J Frankl Institute 352:1836-1849

Najariyan M, Mazandarani M (2015) A note on "Numerical solutions for linear system of first-order fuzzy differential equations with fuzzy constant coefficients". Inform Sci 305:93-96 
Patino M, Nunez MP (2011) Retrofit Approach forthe Reduction of Water and Energy Consumption in Pulp and Paper Production Processes. Environmental Management in Practice, Dr. ElzbietaBroniewicz (Ed). doi:10.5772/20882

Tian Z, Hu L, Greenhalgh D (2010) Perturbation analysis of fuzzy linear systems. Inform Sci 180:4706-4713

Wang C, Xinggan Fu, Meng S, He Y (2017) SPIFGIA operators and their applications to decision making. Granul Comput. doi:10.1007/s41066-016-0025-2
Wang X, Zhong Z, Ha M (2001) Iteration algorithms for solving a system of fuzzy linear equations. Fuzzy Sets Syst 119:121-128

Zadeh LA (1975) The concept of a linguistic variable and its application to approximate reasoning-1. Inf Sci 8:199-249

Zimmermann HJ (2001) Fuzzy set theory and its applications. Springer Business Economics

Zolfaghari ZS, Mohebbi M, Najariyan M (2014) Application of fuzzy linear regression method for sensory evaluation of fried donut. Appl Soft Comput 22:417-423 